\title{
Downregulation of microRNA-124-3p suppresses the mTOR signaling pathway by targeting DDIT4 in males with major depressive disorder
}

\author{
QIULING WANG ${ }^{1}$, GAOFENG ZHAO $^{1}$, ZHENZHEN YANG $^{1}$, XIA LIU $^{1}$ and PING XIE ${ }^{2,3}$ \\ ${ }^{1}$ Department of Psychiatry, Jining Psychiatric Hospital, Jining, Shandong 272051; ${ }^{2}$ Department of Biochemistry \\ and Molecular Biology; ${ }^{3}$ Beijing Key Laboratory for Cancer Invasion and Metastasis Research, \\ Capital Medical University, Beijing 100069, P.R. China
}

Received January 31, 2017; Accepted September 26, 2017

DOI: 10.3892/ijmm.2017.3235

\begin{abstract}
Recent investigations have suggested that microRNAs (miRNAs or miRs) are involved in several pathways that may contribute to the pathomechanism of major depressive disorder (MDD). Sex may not only act as a demographic factor in clinical practive, but may also play a vital role in the molecular heterogeneity of MDD. Although many molecular changes correlated with MDD are found in males, the molecular mechanisms of MDD remain poorly understood. The present study performed bioinformatics analysis to investigate the pathomechanism of MDD in males. The present study identified miR-124-3p as one of the most dysregulated miRNAs in MDD, with decreased expression in the post-mortem BA44 brain area of male patients with MDD. In addition, miR-124-3p targets DNA damage-inducible transcript 4 (DDIT4) and specificity protein 1 (SP1), a DDIT4 transcription factor, in the validated target module of the miRWalk 2.0 database. This is concurrent with an increase in the expression level of DDIT4, which is an inhibitor of the mammalian target of rapamycin (mTOR) signaling pathway. It was also demonstrated that miR-124-3p expression was positively associated with mTOR signaling and this relationship was dependent on the tuberous sclerosis proteins $1 / 2$ complex. Taken together, these results provided a novel insight on miR-124-3p involvement in the biological alterations of male patients with MDD and suggested that this
\end{abstract}

Correspondence to: Professor Ping Xie, Department of Biochemistry and Molecular Biology, Capital Medical University, 10 Xitoutiao, Beijing 100069, P.R. China

E-mail: xpxp922@163.com

Abbreviations: MDD, major depressive disorder; mTOR, mammalian target of rapamycin; DDIT4, DNA damage-inducible transcript 4 protein; TSC1/2, tuberous sclerosis proteins $1 / 2$; ARNT, aryl hydrocarbon receptor nuclear translocator

Key words: major depressive disorder, microRNA-124-3p, DNA damage-inducible transcript 4 , mammalian target of rapamycin signaling, aryl hydrocarbon receptor nuclear translocator
miRNA may also serve as a male-specific target for antidepressant treatment.

\section{Introduction}

Major depressive disorder (MDD) is associated with a high degree of morbidity and mortality (1), and is predicted to be the leading cause of disease burden by the year 2030 (2). MDD occurs about twice as often in women than in men (3) and sex difference is the most obvious characteristics for $\operatorname{MDD}(3,4)$. An increasing number of sex-specific biomarkers for MDD have been identified (5). Moreover, male and female mice have demonstrated fundamentally different transcriptional and post-transcriptional responses to stress, and sex-specific molecular processes occurred following stress (6). Notably, males demonstrated a very robust transcriptional and post-transcriptional response to stress (6). These findings revealed that the pathogenesis of MDD is markedly different in males and females, and transcriptional and post-transcriptional dysregulation may lead to occurrence of MDD in males. Although these changes were found to correlate with MDD in males, the molecular mechanism remains poorly understood.

MicroRNAs (miRNAs or miRs), a prominent class of small non-coding RNAs, generally exert their functions by binding the $3^{\prime}$ untranslated regions of target mRNA to suppress target gene expression in post-transcriptional regulation and control various biological processes (7). The majority of known mammalian miRNA are expressed in a tissue-specific manner, cause translational repression and may individually target hundreds of genes (8). miRNAs are highly expressed in neurons, where they regulate the expression of a large number of target mRNA. Neuronal miRNA pathways may create an extremely powerful mechanism to dynamically adjust the protein content of neuronal compartments, even without the requirement for new gene transcription $(9,10)$. Dysregulation of specific miRNA has been observed in patients and animal models of neuropsychiatric disorders (11-14). In addition, a critical component necessary for miRNA biogenesis, Dicer1, has also been implicated in posttraumatic stress and anxiolytic responses $(15,16)$. With regard to MDD, the evidence supporting miRNA involvement in the pathophysiology and the treatment of the disorder is 
increasing (17). In addition, numerous studies have implied that miRNA may provide a link between environmental stressors and gene expression (18), and be an important factor involved in the mechanism of depression (19-22). In vertebrates, more distinct miRNAs are expressed in the brain than in any other tissues $(23,24)$. These studies indicate that miRNA may serve an important role in the brain of patients with MDD by acting as the master regulator of gene expression at a post-transcriptional level (25-27). Previous investigations have suggested that miRNA have been involved in several pathways that may contribute to the MDD pathomechanism (27-29). However, how these miRNA contribute to this illness is not well understood.

The present study identified that miR-124-3p, an abundant miRNA in the brain, was significantly downregulated in the post-mortem brain BA44 area of males with MDD. The miR-124-3p target gene, DNA damage-inducible transcript 4 (DDIT4), a regulator of the tuberous sclerosis proteins $1 / 2(\mathrm{TSC} 1 / 2)$ complex, was associated with MDD pathogenesis and antidepressant effect. Meanwhile, the expression of miR-124-3p was negatively correlated with expression of DDIT4 and DDIT4 transcription factor, specificity protein 1 (SP1), respectively. miR-124-3p regulated the mammalian target of rapamycin (mTOR) pathway significantly and this regulation was dependent on the TSC1/2 complex. In addition, miR-124-3p was downregulated by reduction of its transcription factor, aryl hydrocarbon receptor nuclear translocator (ARNT), in males with MDD. These results may help to elucidate the pathogenesis of MDD and provide novel insight into antidepressant treatment.

\section{Materials and methods}

Gene Expression Omnibus (GEO) dataset collection and differential expression analysis. Microarray data were obtained from five datasets. The five series were accessed at the National Centers for Biotechnology Information GEO database (ncbi.nlm.nih.gov/geo/), which served as a public repository for gene expression datasets, and the accession numbers were GSE58105, GSE63377, GSE43261, GSE64119 and GSE22909, respectively. Differentially expressed genes and miRNAs were obtained using GEO2R (ncbi.nlm.nih. gov/geo/geo $2 \mathrm{r} /$ ). GEO2R is an interactive web tool that compares two groups of samples under the same experimental conditions and is able to analyze almost any GEO series (30).

miRNA tissue-specific expression analysis. Expression levels of miR-124-3p across human tissues were obtained from the YM500 database (http://ngs.ym.edu.tw/ym500v2/index.php).

Prediction of target genes of miRNA. The miRWalk 2.0 database (zmf.umm.uni-heidelberg.de/apps/zmf/mirwalk2/) (31) was used to identify genes that are directly targeted by miR-124-3p in the validated target module.

The Cancer Genome Atlas (TCGA) data exploratory analyses. To determine whether the expression level of DDIT4 was correlated with miR-124-3p in brain tissues, TCGA data about miRNA and mRNA (RNA Seq v2) expression levels in patients with low-grade glioma were obtained from synapse. org/ for correlation analysis in a large data set $(n=528)$. The
miR-124 level, DDIT4 mRNA level, SP1 mRNA level and whole transcriptome data were used in the present study.

Transcription factor analysis and target gene binding site prediction. The GeneCards database (genecards.org/), JASPAR database (jaspar.binf.ku.dk/) and PROMO database (alggen.lsi.upc.es/cgi-bin/promo_v3/promo/promoinit. cgi?dirDB=TF_8.3) were used to identify potential transcription factor-gene interactions. The GeneCards database was used to investigate whether SP1, a target of miR-124-3p, is a transcription factor of DDIT4, and whether it regulates DDIT4 expression. The binding sequence and binding sites were shown in the promoter region of DDIT4. To investigate the cause of miR-124-3p downregulation, potential transcription factors, which are also downregulated in males with MDD according to the results of gene ontology (GO) analyses, were first screened. Subsequently, the JASPAR and PROMO databases were used to predict transcription factors of miR-124.

Gene set enrichment analysis. The association between phenotypes, pathways and miR-124-3p expression was analyzed using Gene Set Enrichment Analysis (GSEA v2.2; broad.mit.edu/gsea/). GSEA calculates a gene set enrichment score (ES) that estimates whether genes from a pre-defined gene set (obtained from the Molecular Signatures Database; http://software.broadinstitute.org/gsea/msigdb/collections.jsp\#C2; MTOR_UP.N4.V1_DN, BILANGES_RAPAMYCIN_ SENSITIVE_VIA_TSC1_AND_TSC2) are enriched among the highest- (or lowest-) ranked genes or distributed randomly. Default settings were used. Thresholds for significance were determined by permutation analysis (1,000 permutations). False discovery rate (FDR) was calculated. A gene set was considered significantly enriched when the FDR score was $<0.05$.

GO analyses. A list of differentially expressed genes in males with MDD was imported into the Database for Annotation, Visualization, and Integrated Discovery Bioinformatics Resource (david.abcc.ncifcrf.gov/). The aim was to detect significantly over-represented biological processes affected by changes in the transcriptomes. Functional enrichment was assessed using the following three annotation databases: Biological process, molecular function and cellular component.

Statistical analysis. Statistical analyses were performed using SPSS 19.0 (IBM Corp., Armonk, NY, USA), R 3.2.4 software (r-project.org/), and GraphPad Prism 5 (GraphPad Software, Inc., La Jolla, CA, USA). The data were presented as the mean \pm standard deviation. Group distributions were compared using the Student's t-test. For multiple comparisons, one-way analysis of variance was used followed by Dunnett's post hoc test. The correlation between miR-124-3p and target gene expression level was analyzed by Pearson correlation analysis. $\mathrm{P}<0.05$ was considered to indicate a statistically significant difference.

\section{Results}

Expression level of miR-124-3p is downregulated in males with $M D D$. As we know, age and sex are complicated factors when trying to understand the pathomechanism of MDD $(4,32,33)$. In 

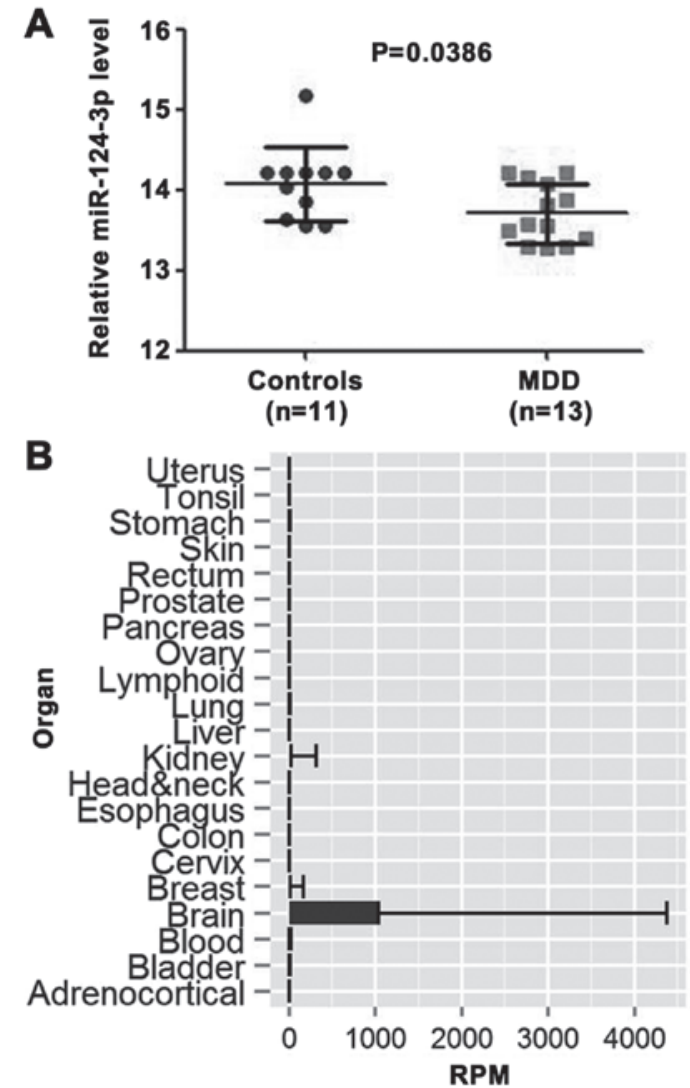

Figure 1. Expression levels of miR-124-3p. (A) Expression levels of miR-124-3p in the BA44 area of post-mortem brain samples of human patients with MDD and control subjects (GSE58105). (B) Expression of miR-124-3p across human tissues (YM500v2 database). RPM, reads per million reads; miR, microRNA.

consideration of this, the data in the GEO miRNA expression profile (GSE58105) were filtered, and 24 young male samples (MDD, $n=13$; controls, $n=11$ ) were selected, according to previous studies $(32,34)$. Differential expression analysis was performed between the prefrontal cortex (PFC) of depressed males and healthy controls using GEO2R. This result demonstrated that the miR-124-3p expression level was significantly higher in control samples than in MDD samples $(\mathrm{P}=0.0386$; Fig. 1A). A total of five differentially expressed miRNA (has-miR-423-5p, -miR-320c, -miR-124, -miR-1825 and -miR-1281) were screened under the criteria $(\mathrm{P}<0.05 ; \mathrm{FC}>1.2$ or FC<0.8). Subsequently, multiple testing was also performed and the FDR of candidate differential expression miRNA was calculated using R 3.2.4. The FDR of miR-124 was also $<0.05$ (data not shown). According to tissue specificity analysis from the YM500 database, miR-124-3p was the only miRNA enriched in the brain (Fig. 1B). Therefore, it was hypothesized that the downregulation of miR-124-3p may play an important role in the occurrence of MDD.

DDIT4 and its transcription factor, SPI, are targets of $m i R-124-3 p$. To investigate the mechanism of miR-124-3p in the occurrence of MDD, the validated target genes of miR-124-3p from the miRWalk 2.0 database were examined. DDIT4 (REDD1), a target of miR-124-3p (data not shown), was reported to act as a critical mediator in MDD (35). To further verify the role of DDIT4, microarray profiles GSE63377 and GSE43261 were used. As demonstrated in Fig. 2A, the DDIT4 mRNA level was upregulated in rats with depressive-like behavior. In male mice, the DDIT4 mRNA level was significantly downregulated in fluoxetine sensitivity group compared with fluoxetine resistance group and control group, respectively $(\mathrm{P}=0.011$ and $\mathrm{P}<0.0001$, respectively; Fig. 2B). These data revealed the role of DDIT4 in the occurrence and treatment effect of MDD.

In a previous study, depression also acted as a risk factor in patients with low-grade glioma (36). It has been proposed that depression is not only a psychological reaction to the low-grade glioma, but that the association between these disorders has biochemical roots (36-38). To further determine whether the expression level of DDIT4 was regulated by miR-124-3p, correlation analyses between the expression level of DDIT4 and miR-124-3p were performed in low-grade glioma patients. This result demonstrated that the expression level of miR-124-3p was negatively correlated with the expression level of DDIT4 (Fig. 2C; $\mathrm{R}^{2}=0.02 ; \mathrm{P}=0.0011$ ). In addition, the expression level of miR-124-3p was negatively correlated with the expression level of SP1, the other validated target gene of miR-124-3p (Fig. 2D; $\mathrm{R}^{2}=0.13 ; \mathrm{P}<0.001$ ). Notably, SP1, a transcription factor, could bind to the transcription start site of DDIT4 (Fig. 2E). To validate whether SP1 is able to regulate the expression of DDIT4, microarray profile GSE64119 was used. As indicated in Fig. 2F, DDIT4 was significantly downregulated by MitA $(\mathrm{P}<0.05)$, which is a direct inhibitor of the binding of SP1 family factors to DNA promoters (39). These data suggested that miR-124-3p suppresses the expression of DDIT4 by targeting DDIT4 and its transcription factor SP1.

Regulatory effects of miR-124-3p on the mTOR signaling pathway are dependent on the TSC1/2 complex. DDIT4, a target gene of miR-124-3p, is a well-known antagonist of mTOR signaling in MDD (35). The mTOR signaling pathway serves an important role in MDD (35), therefore, to investigate the regulatory effect of miR-124-3p on mTOR signaling, GSEA was used. The patients with low-grade glioma from TCGA were divided into miR-124-3p-positive and miR-124-3p-negative groups, and the association between miR-124-3p and mTOR signaling was analyzed. As demonstrated in Fig. 3A, the mTOR activation gene set was significantly enriched in the miR-124-3p-positive group ( $F D R=0.02)$. These data suggested that the miR-124-3p expression level was positively associated with mTOR signaling. To further verify the regulation of miR-124-3p on mTOR signaling by targeting DDIT4, which stabilizes the TSC1/2 complex and inhibits the mTOR signaling, GSEA was performed and a mTOR pathway activation gene set was employed, which was obtained from TSC1(-/-) or TSC2(-/-) mouse embryo fibroblasts. The miR-124-3-positive samples were divided into high and low miR-124-3p expression groups. As demonstrated in Fig. 3B, this mTOR pathway gene set was not significantly enriched in each group. These results suggested that the regulatory effect of miR-124-3p on mTOR signaling is dependent on the TSC1/2 complex. Thus, miR-124-3p may play a role in the regulation of mTOR signaling by targeting DDIT4.

Microarray-based GO analyses. From the GEO mRNA expression profile (GSE53987), differentially expressed mRNAs were identified between the PFC of depressed males and normal control samples. To gain insights into the biolog- 
A
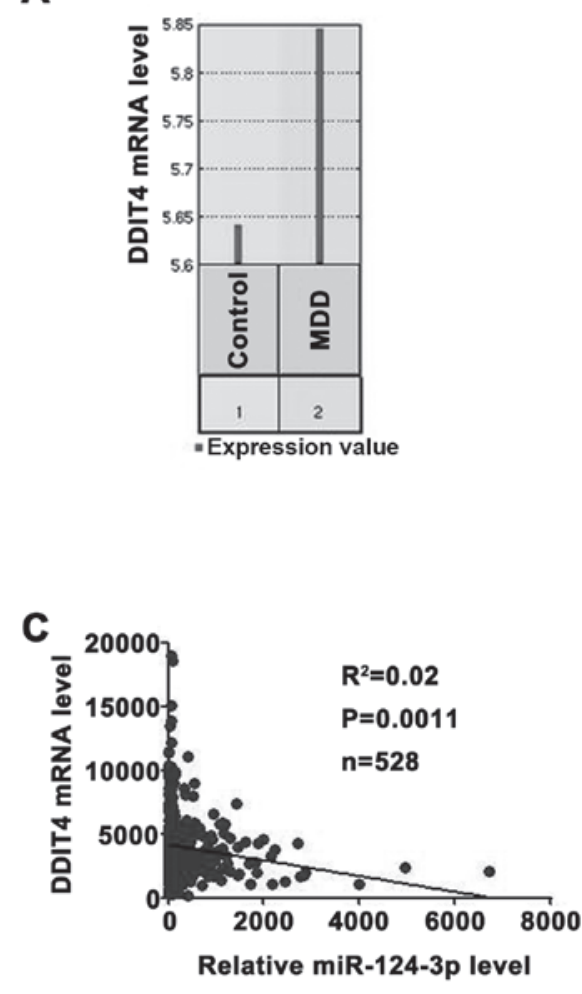

E

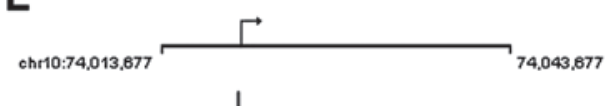

\begin{tabular}{|c|c|c|}
\hline Binding Position & Strand & Binding Sequence \\
\hline chr10: 74033508-74033521 & + & ATGGAGGCGGGGCC \\
\hline \multicolumn{3}{|l|}{ Legend: } \\
\hline \multicolumn{3}{|l|}{$\Gamma$ Transcription start site of DDIT4 } \\
\hline
\end{tabular}

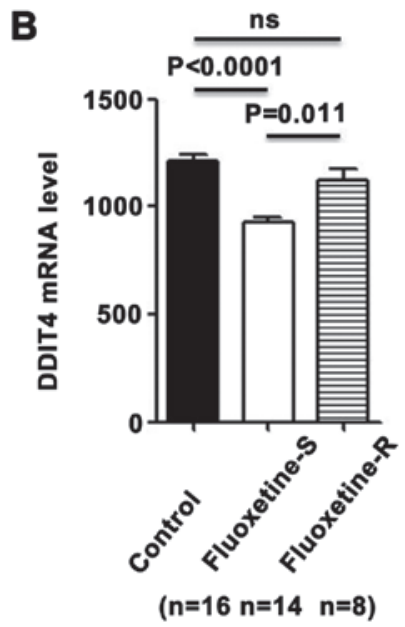

D

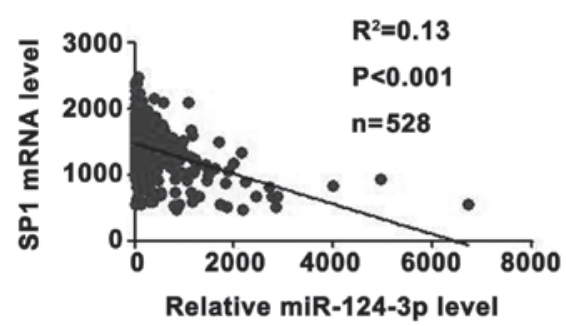

$\mathbf{F}$

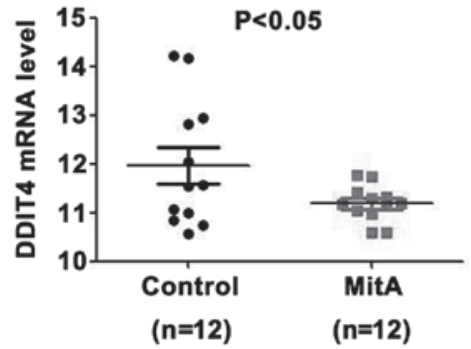

Figure 2. miR-124-3p inhibits expression of DDIT4 by directly targeting DDIT4 and its transcription factor, SP1. (A) Downregulation of the DDIT4 expression level in the PFC of male rats with depressive-like behavior (GSE63377). (B) Relative expression level of miR-124-3p in the PFC of male mice. The expression level of DDIT4 was reduced in the fluoxetine-S group compared with the level in the fluoxetine-R group and control group, respectively (GSE43261). Significance was determined with one-way analysis of variance followed by Dunnett's post hoc test. (C) miR-124-3p expression level is negatively correlated with DDIT4 mRNA expression level, as demonstrated in 528 patients with low-grade glioma from TCGA dataset. (D) miR-124-3p expression level is negatively correlated with SP1 mRNA expression level, as demonstrated in 528 patients with low-grade glioma from TCGA dataset. (E) SP1 is a potential transcription factor of DDIT4. The arrow indicates the transcription start site of DDIT4 in the chromosome position. The vertical line is the SP1 binding site. The binding position of SP1 on the chromosome and the binding sequence are shown in the table. (F) MitA, a direct inhibitor of the binding of SP1 family factors to DNA promoters, suppressed the expression of DDIT4 (GSE64119). Significance between the two populations was determined with a two-tailed t-test. PFC, prefrontal cortex; ns, not significant; TCGA, The Cancer Genome Atlas; miR, microRNA; DDIT4, DNA damage-inducible transcript 4 protein; MDD, major depressive disorder; fluoxetine-S, fluoxetine-sensitive; fluoxetine-R, fluoxetine-resistant.

ical roles of differently expressed genes, we performed GO classification enrichment analysis. Genes that demonstrated a nominal significance level of $\mathrm{P}<0.01$ were selected and were tested against the background set of all genes with $\mathrm{GO}$ annotations. As indicated in Fig. 4, the most enriched GO categories of the differentially expressed genes were 'development process', 'nucleic acid binding transcription factor activity', 'transcription factor complex' and 'nucleus.' According to these results, the regulation of transcription factors was associated with the occurrence of MDD in males.

ARNT regulates the expression of miR-124-3p by acting as its transcription factor. According to the GO analysis results, it was hypothesized that the expression of miR-124-3p was regulated by its transcription factor in this disease. Among the 38 transcription factors, 25 were downregulated in males with MDD (data not shown). Transcription factors of miR-124-3p were predicted from the JASPAR and PROMO databases. As demonstrated in Fig. 5A, ARNT may be the transcription factor that has a role in MDD. To further investigate the regulation of ARNT on miR-124-3p expression, microarray profile GSE22909 was used. As indicated in Fig. 5B, miR-124-3p expression was upregulated by 2,3,7,8-tetrachlorodibenzo-p-dioxin, which is an activator for ARNT $(40,41)$. These results suggested that miR-124-3p was downregulated by the reduction of ARNT in males with MDD. As demon- 

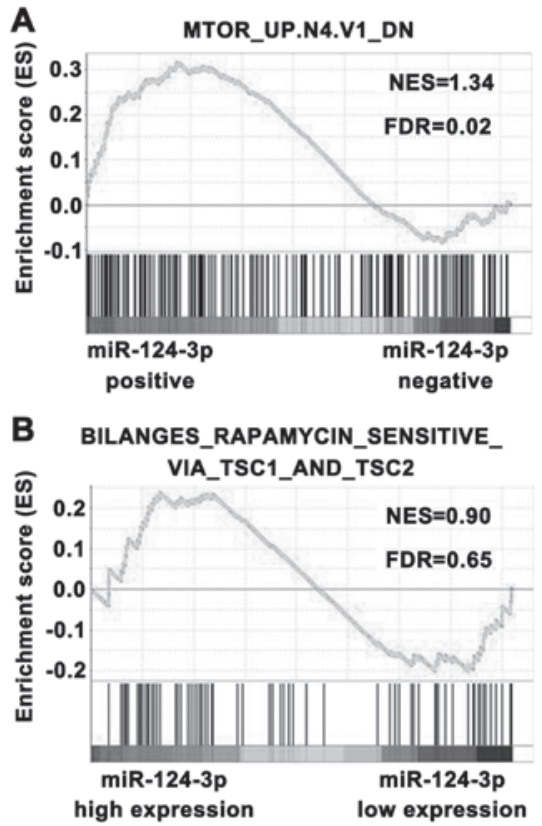

Figure 3. miR-124-3p is positively associated with mTOR signaling and TSC1/2 knockout abolished its regulatory effect on mTOR signaling. (A) miR-124-3p is positively associated with mTOR activation. GSEA of TCGA data depicting the enrichment of the mTOR pathway activation gene set. Samples were divided into miR-124-3p-positive and -negative groups. The mTOR activation gene set was enriched in the miR-124-3p-positive group. (B) The regulation of miR-124-3p on the mTOR signaling pathway is dependent on the TSC1/2 complex. GSEA of TCGA data depicting the enrichment of the mTOR pathway activation gene set, which was obtained from TSC1(-/-) or TSC2(-/-) mouse embryo fibroblasts. miR-124-3p expression was not significantly associated with this gene set. miR-124-3p-positive samples were divided into high and low miR-124-3p expression groups. miR, microRNA; mTOR, mammalian target of rapamycin; $\mathrm{TSC} 1 / 2$, tuberous sclerosis proteins 1/2; GSEA, Gene Set Enrichment Analysis; TCGA, The Cancer Genome Atlas; NES, normalized enrichment score; FDR, false discovery rate.

A

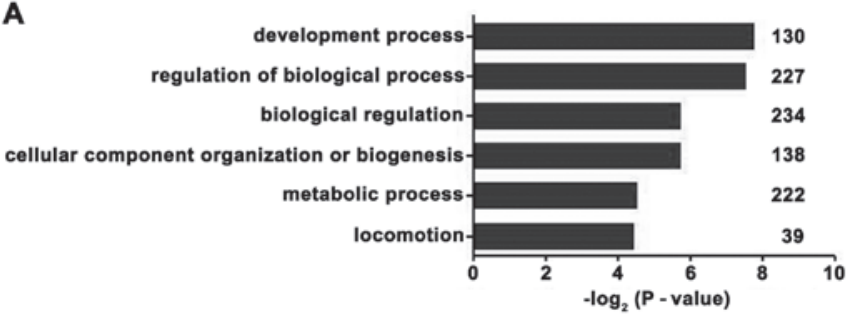

B

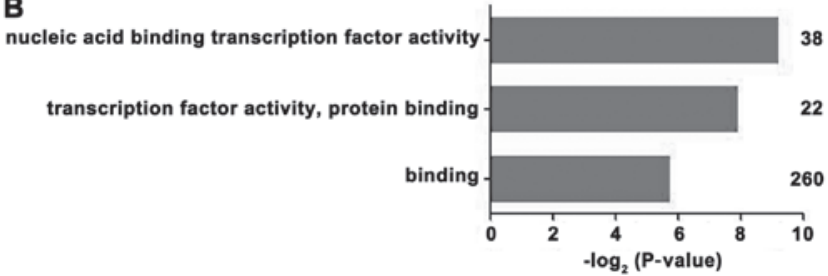

C

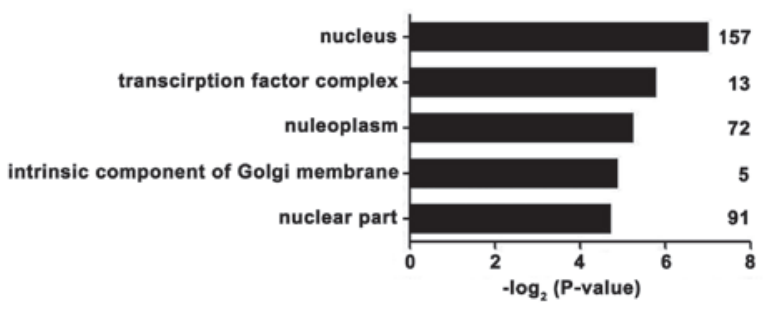

Figure 4. GO analysis for differential expression of genes in males with major depressive disorder. GO analysis of the differentially expressed genes according to (A) biological process, (B) molecular function and (C) cellular component. The numbers are counts involved in GO analysis results. GO, gene ontology.

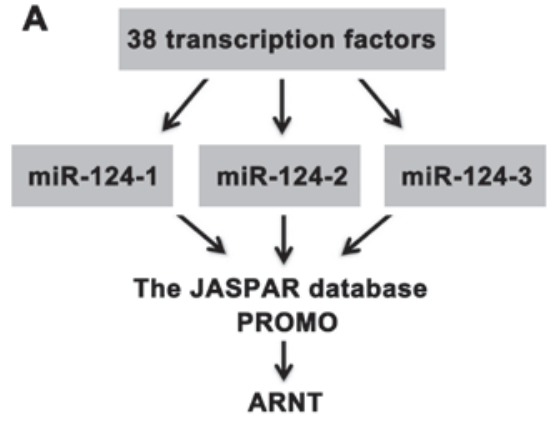

B

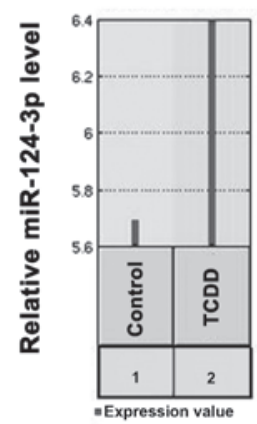

Figure 5. Expression of miR-124-3p is regulated by its transcription factor, ARNT. (A) Flow chart of screening for the main transcription factors of miR-124-3p in males with MDD. A total of 38 transcription factors were obtained from gene ontology analysis results on biological processes. ARNT was the main transcription factor of miR-124-3p in males with MDD. (B) TCDD (an activator for the transcription factor aryl hydrocarbon receptor/ARNT) increased the expression of miR-124-3p (GSE22909). miR, microRNA; ARNT, aryl hydrocarbon receptor nuclear translocator; MDD, major depressive disorder; TCDD, 2,3,7,8-tetrachlorodibenzo-p-dioxin.

strated in Fig. 6, ARNT downregulation reduced miR-124-3p expression. miR-124-3p downregulation may result in mTOR signaling inhibition and occurrence of MDD in males via regulating DDIT4 expression. miR-124-3p may regulate DDIT4 expression by targeting DDIT4 and SP1.

\section{Discussion}

Emerging evidences have suggested that miRNA may serve a key role in the basic mechanisms of brain neuroplasticity, and they are believed to be involved in the manifestation of depression and the therapeutic actions of antidepressant drugs (42). A study by Lopez et al (25) demonstrated BA44 microarray expression of miRNA in MDD. In the present study, it was demonstrated that miR-124-3p was one of most significantly dysregulated miRNA in the BA44 microarray of Lopez et al (25). To the best of our knowledge, the present study is the first to report the downregulation of miR-124-3p in males with MDD.

In the present study, the results indicated that miR-124-3p was downregulated in patients with MDD compared with healthy controls. To determine the role and mechanism of miR-124-3p in MDD, bioinformatics analysis was used. As a result, it was identified that miR-124-3p was downregulated in patients with MDD. DDIT4, a validated target gene of miR-124-3p, was correlated with the occurrence of MDD and antidepressant effect. miR-124-3p reduced DDIT4 expression by targeting DDIT4 and DDIT4 transcription factor SP1, which led to the regulation of the mTOR signaling pathway. In addi- 


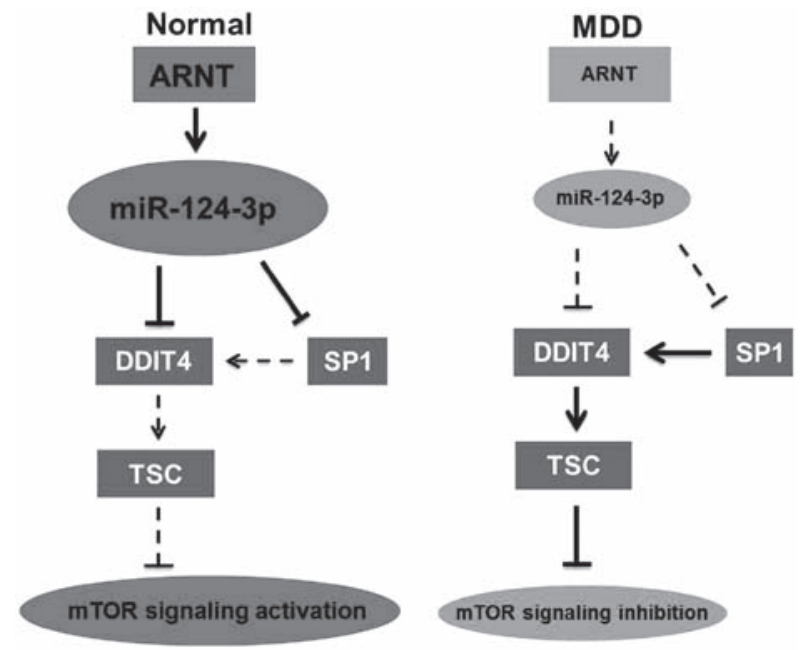

Figure 6. Model for the potential mechanism by which reduced miR-124-3p contributes to the occurrence of MDD in males. Schematic showing how reduced miR-124-3p could result in decreased mTOR signaling and occurrence of disease via regulating DDIT4 expression. In individuals without MDD, miR-124-3p inhibits expression of DDIT4 and promotes activation of the mTOR signaling pathway. In MDD, downregulation of miR-124-3p abolishes its inhibition of DDIT4 expression and inhibits the mTOR signaling pathway. miR, microRNA; MDD, major depressive disorder; mTOR, mammalian target of rapamycin; DDIT4, DNA damage-inducible transcript 4 protein; ARNT, aryl hydrocarbon receptor nuclear translocator; TSC, tuberous sclerosis proteins.

tion, decreased ARNT levels resulted in reduced miR-124-3p levels in males with MDD.

Previous studies have reported that miR-124 may regulate adult neurogenesis, promote neuronal differentiation and contribute to synaptic plasticity in vivo $(5,43)$. Although miR-124 has been widely studied in animal models of depression, few studies have investigated the relationship between the levels of miR-124 and MDD in clinics. Recent studies have reported that miR-124-3p was upregulated in the BA46 area of the brain (44) and in peripheral blood mononuclear cells in patients with MDD (45). miR-124 expression levels were also increased in the hippocampus of rat models of depression $(46,47)$. Contrastingly, two previous studies did not find altered levels of miR-124 $(34,48)$. However, to the best of our knowledge, the present study is the first to identify that miR-124-3p was downregulated in the BA44 brain area in patients with MDD, although the fold-change of miR-124-3p was not tremendous. Notably, minor fold-change of critical mediators in a signaling pathway usually have a cascade amplification effect on occurrence of disease, such as subtle reductions in the dose of phosphatase and tensin homolog predispose to tumorigenesis (49). In the present study, the downregulation of miR-124-3p contributed to the inhibition of the mTOR signaling pathway, which serves an important role in MDD. Therefore, miR-124-3p may have a cascade amplification effect in the occurrence of MDD by regulating DDIT4 expression and mTOR signaling, and so the fold-change is not the only guide for gene function in the occurrence of disease. The minor downregulation of miR-124-3p may have stronger biological effects than other notable differentially expressed genes. In addition, the search for the main genetic effects in MDD so far has not revealed consistent or replicated signifi- cant findings (50), implying that it is difficult to identify the main causative genes in MDD, and so the weak expression differences may be an important feature of MDD.

In the present study, there were limited numbers of controls and MDD subjects in the GEO database. Perhaps, it is difficult to collect post-mortem brain tissues. Furthermore, the incidence of MDD is relatively low in the population (51), which makes it harder to collect data on patients with MDD. Efforts should be made to collect more samples in follow-up work.

Previous studies have reported decreased levels of the mTOR complex 1 (mTORC1) signaling pathway in post-mortem PFC samples of subjects with MDD (52) and mTOR signaling was correlated with neuronal brain function in MDD (50). Notably, DDIT4, a critical mediator of the mTOR signaling pathway in MDD, was a validated target gene of miR-124-3p. In addition, SP1, which is a transcription factor of DDIT4, was also a validated target gene of miR-124-3p. miR-124-3p may regulate expression of DDIT4 by directly targeting DDIT4 and SP1, which is a transcription factor of DDIT4. To further investigate the relationship between the mTORC1 signaling pathway and miR-124-3p, GSEA was performed. The present results indicated that the miR-124-3p expression level was positively associated with activation of mTOR pathway. In addition, the regulatory effect of miR-124-3p on the mTOR signaling pathway is dependent on the TSC1/2 complex. These results revealed that miR-124-3p may regulate the mTOR signaling pathway by reducing the expression of DDIT4, which stabilizes the complex formed by TSC1 and TSC2; this complex subsequently inhibits mTORC1-dependent protein synthesis and cell growth (50). Research has demonstrated that miR-124-3p was correlated with cell growth, proliferation and apoptosis, as well as the mTOR pathway (45). Diagnostically, MDD is associated with smaller regional brain volumes (53). In addition, the mTOR pathway serves an important role in neurodevelopment of the cerebral neocortex and neurodevelopmental diseases (54). Similarly, the results of the present study indicated that the most aberrant biological process is the development process in males with MDD. These results suggested that miR-124-3p may have an important role in these biological processes.

In a previous study, it was reported that two $\mathrm{CpG}$ islands were identified in the miR-124-3 gene promoter, and that the promoter methylation level determined the miR-124-3p expression level in corticosterone-treated rats (44). The present results concerning the human miR-124-3 gene promoter $\mathrm{CpG}$ islands were different to the finding in this previous study. Similarly, we used MethPrimer (urogene.org/methprimer/) to predict CpG islands of miR-124-3 gene promoter in humans. No $\mathrm{CpG}$ islands were identified in the human miR-124-3 gene promoter (data not shown). According to the present GO analysis results, the majority of differentially expressed genes were transcription factors or had transcription factor activity, suggesting that downregulation of some transcription factors may be one of the prime reasons of miR-124-3p downregulation. Further bioinformatics analysis revealed that ARNT was a potential transcription factor that could regulate the expression of miR-124. To the best of our knowledge, this is a novel mechanistic study on the regulation of miR-124 expression.

In conclusion, the results of the present study suggested that miR-124-3p was involved in the pathogenesis and treat- 
ment of MDD in males via regulation of DDIT4 expression and the mTOR signaling pathway. Reduced expression levels of miR-124-3p resulted in a reduced ARNT level in MDD. These findings provide novel insight into the mechanism of MDD occurrence and potential antidepressant treatment.

\section{Acknowledgments}

The present study was supported by the Chinese National Natural Science Foundation Projects (grant no. 31670774), Beijing Nova Program (grant no. Z161100004916059) and Support Project of High-level Teachers in Beijing Municipal Universities in the Period of 13th Five-year Plan (grant no. CIT\&TCD201704097).

\section{References}

1. Merikangas KR, Akiskal HS, Angst J, Greenberg PE Hirschfeld RM, Petukhova M and Kessler RC: Lifetime and 12-month prevalence of bipolar spectrum disorder in the National Comorbidity Survey replication. Arch Gen Psychiatry 64: 543-552, 2007.

2. World Health Organization: Global burden of disease 2004 update: Disability weights for diseases and conditions. WHO, Geneva, Switzerland, 2004. Available at: http://www.who.int/healthinfo/global_burden_ disease/GBD2004 DisabilityWeights.pdf.

3. Seedat S, Scott KM, Angermeyer MC, Berglund P, Bromet EJ, Brugha TS, Demyttenaere K, de Girolamo G, Haro JM, Jin R, et al: Cross-national associations between gender and mental disorders in the World Health Organization World Mental Health Surveys. Arch Gen Psychiatry 66: 785-795, 2009.

4. Otte C, Gold SM, Penninx BW, Pariante CM, Etkin A, Fava M, Mohr DC and Schatzberg AF: Major depressive disorder. Nat Rev Dis Primers 2: 16065, 2016.

5. Cheng LC, Pastrana E, Tavazoie M and Doetsch F: miR-124 regulates adult neurogenesis in the subventricular zone stem cell niche. Nat Neurosci 12: 399-408, 2009.

6. Pfau ML, Purushothaman I, Feng J, Golden SA, Aleyasin H, Lorsch ZS, Cates HM, Flanigan ME, Menard C, Heshmati M, et al: Integrative analysis of sex-specific microRNA networks following stress in mouse nucleus accumbens. Front Mol Neurosci 9: 144, 2016.

7. Krol J, Loedige I and Filipowicz W: The widespread regulation of microRNA biogenesis, function and decay. Nat Rev Genet 11: 597-610, 2010.

8. He L and Hannon GJ: MicroRNAs: Small RNAs with a big role in gene regulation. Nat Rev Genet 5: 522-531, 2004

9. Schratt G: Fine-tuning neural gene expression with microRNAs. Curr Opin Neurobiol 19: 213-219, 2009.

10. Ul Hussain M: Micro-RNAs (miRNAs): Genomic organisation, biogenesis and mode of action. Cell Tissue Res 349: 405-413, 2012.

11. Forero DA, van der Ven K, Callaerts P and Del-Favero J: miRNA genes and the brain: Implications for psychiatric disorders. Hum Mutat 31: 1195-1204, 2010.

12. Miller BH and Wahlestedt C: MicroRNA dysregulation in psychiatric disease. Brain Res 1338: 89-99, 2010.

13. Moreau MP, Bruse SE, David-Rus R, Buyske $S$ and Brzustowicz LM: Altered microRNA expression profiles in postmortem brain samples from individuals with schizophrenia and bipolar disorder. Biol Psychiatry 69: 188-193, 2011.

14. Chan AW and Kocerha J: The Path to microRNA therapeutics in psychiatric and neurodegenerative disorders. Front Genet 3: 82, 2012.

15. Dias C, Feng J, Sun H, Shao NY, Mazei-Robison MS, Damez-Werno D, Scobie K, Bagot R, LaBonté B, Ribeiro E, et al: $\beta$-catenin mediates stress resilience through Dicer1/microRNA regulation. Nature 516: 51-55, 2014.

16. Wingo AP, Almli LM, Stevens JS and Klengel T, Uddin M, Li Y, Bustamante AC, Lori A, Koen N and Stein DJ: DICER1 and microRNA regulation in post-traumatic stress disorder with comorbid depression. Nat Commun 6: 10106, 2015.
17. Dwivedi Y: Pathogenetic and therapeutic applications of microRNAs in major depressive disorder. Prog Neuropsychopharmacol Biol Psychiatry 64: 341-348, 2016.

18. Kuss AW and Chen W: MicroRNAs in brain function and disease. Curr Neurol Neurosci Rep 8: 190-197, 2008.

19. Dwivedi Y: Evidence demonstrating role of microRNAs in the etiopathology of major depression. J Chem Neuroanat 42: 142-156, 2011.

20. Ha TY: MicroRNAs in human diseases: From autoimmune diseases to skin, psychiatric and neurodegenerative diseases. Immune Netw 11: 227-244, 2011.

21. Mouillet-Richard S, Baudry A, Launay JM and Kellermann O: MicroRNAs and depression. Neurobiol Dis 46: 272-278, 2012.

22. Smalheiser NR, Lugli G, Rizavi HS, Torvik VI, Turecki G and Dwivedi Y: MicroRNA expression is down-regulated and reorganized in prefrontal cortex of depressed suicide subjects. PLoS One 7: e33201, 2012.

23. Fineberg SK, Kosik KS and Davidson BL: MicroRNAs potentiate neural development. Neuron 64: 303-309, 2009.

24. Lagos-Quintana M, Rauhut R, Yalcin A, Meyer J, Lendeckel W and Tuschl T: Identification of tissue-specific microRNAs from mouse. Curr Biol 12: 735-739, 2002.

25. Lopez JP, Lim R, Cruceanu C, Crapper L, Fasano C, Labonte B, Maussion G, Yang JP, Yerko V, Vigneault E, et al:: miR-1202 is a primate-specific and brain-enriched microRNA involved in major depression and antidepressant treatment. Nat Med 20: 764-768, 2014.

26. Xu Y, Liu H, Li F, Sun N, Ren Y, Liu Z, Cao X, Wang Y, Liu P and Zhang K: A polymorphism in the microRNA-30e precursor associated with major depressive disorder risk and P300 waveform. J Affect Disord 127: 332-336, 2010.

27. Saus E, Soria V, Escaramís G, Vivarelli F, Crespo JM, Kagerbauer B, Menchón JM, Urretavizcaya M, Gratacòs M and Estivill X: Genetic variants and abnormal processing of pre-miR-182, a circadian clock modulator, in major depression patients with late insomnia. Hum Mol Genet 19: 4017-4025, 2010.

28. Meerson A, Cacheaux L, Goosens KA, Sapolsky RM, Soreq H and Kaufer D: Changes in brain MicroRNAs contribute to cholinergic stress reactions. Journal of molecular neuroscience: MN 40: 47-55, 2010.

29. Vreugdenhil E, Verissimo CS, Mariman R, Kamphorst JT, Barbosa JS, Zweers T, Champagne DL, Schouten T, Meijer OC, de Kloet ER and Fitzsimons CP: MicroRNA 18 and 124a down-regulate the glucocorticoid receptor: Implications for glucocorticoid responsiveness in the brain. Endocrinology 150: 2220-2228, 2009.

30. Barrett T, Wilhite SE, Ledoux P, Evangelista C, Kim IF, Tomashevsky M, Marshall KA, Phillippy KH, Sherman PM, Holko M, et al: NCBI GEO: Archive for functional genomics data sets--update. Nucleic Acids Res 41: D991-D995, 2013.

31. Dweep H, Sticht C, Pandey P and Gretz N: miRWalk--database: Prediction of possible miRNA binding sites by 'walking' the genes of three genomes. J Biomed Inform 44: 839-847, 2011.

32. Kaufman J, Sullivan GM, Yang J, Ogden RT, Miller JM, Oquendo MA, Mann JJ, Parsey RV and DeLorenzo C: Quantification of the serotonin 1A receptor using PET: Identification of a potential biomarker of major depression in males. Neuropsychopharmacology 40: 1692-1699, 2015.

33. Zheng P, Chen JJ, Zhou CJ, Zeng L, Li KW, Sun L, Liu ML, Zhu D, Liang ZH and Xie P: Identification of sex-specific urinary biomarkers for major depressive disorder by combined application of NMR- and GC-MS-based metabonomics. Transl Psychiatry 6: e955, 2016.

34. Belzeaux R,Bergon A,Jeanjean V,Loriod B,Formisano-Tréziny C, Verrier L, Loundou A, Baumstarck-Barrau K, Boyer L, Gall V, et al: Responder and nonresponder patients exhibit different peripheral transcriptional signatures during major depressive episode. Transl Psychiatry 2: e185, 2012.

35. Ota KT, Liu RJ, Voleti B, Maldonado-Aviles JG, Duric V, Iwata M, Dutheil S, Duman C, Boikess S, Lewis DA, et al: REDD1 is essential for stress-induced synaptic loss and depressive behavior. Nat Med 20: 531-535, 2014.

36. Mainio A, Tuunanen S, Hakko H, Niemelä A, Koivukangas J and Räsänen P: Decreased quality of life and depression as predictors for shorter survival among patients with low-grade gliomas: A follow-up from 1990 to 2003. Eur Arch Psychiatry Clin Neurosci 256: 516-521, 2006.

37. Spiegel D and Giese-Davis J: Depression and cancer: Mechanisms and disease progression. Biol Psychiatry 54: 269-282, 2003. 
38. Horrobin DF and Bennett CN: Depression and bipolar disorder: Relationships to impaired fatty acid and phospholipid metabolism and to diabetes, cardiovascular disease, immunological abnormalities, cancer, ageing and osteoporosis. Possible candidate genes. Prostaglandins Leukot Essent Fatty Acids 60: 217-234, 1999.

39. Seznec J, Silkenstedt B and Naumann U: Therapeutic effects of the Sp1 inhibitor mithramycin A in glioblastoma. J Neurooncol 101: 365-377, 2011.

40. Elyakim E, Sitbon E, Faerman A, Tabak S, Montia E, Belanis L, Dov A, Marcusson EG, Bennett CF, Chajut A, et al: hsa-miR-191 is a candidate oncogene target for hepatocellular carcinoma therapy. Cancer Res 70: 8077-8087, 2010

41. Nebert DW, Dalton TP, Okey AB and Gonzalez FJ: Role of aryl hydrocarbon receptor-mediated induction of the CYP1 enzymes in environmental toxicity and cancer. J Biol Chem 279: 23847-23850, 2004.

42. Song MF, Dong JZ, Wang YW, He J, Ju X, Zhang L, Zhang YH, Shi JF and Lv YY: CSF miR-16 is decreased in major depression patients and its neutralization in rats induces depression-like behaviors via a serotonin transmitter system. J Affect Disord 178: 25-31, 2015.

43. Makeyev EV, Zhang J, Carrasco MA and Maniatis T: The MicroRNA miR-124 promotes neuronal differentiation by triggering brain-specific alternative pre-mRNA splicing. Mol Cell 27: 435-448, 2007.

44. Roy B, Dunbar M, Shelton RC and Dwivedi Y: Identification of MicroRNA-124-3p as a putative epigenetic signature of major depressive disorder. Neuropsychopharmacology 42: 864-875, 2017.

45. Bondarenko EA, Shadrina MI, Grishkina MN, Druzhkova TA, Akzhigitov RG, Gulyaeva NV, Guekht AB and Slominsky PA: Genetic analysis of BDNF, GNB3, MTHFR, ACE and APOE variants in major and recurrent depressive disorders in Russia. Int J Med Sci 13: 977-983, 2016.

46. He S, Liu X, Jiang K, Peng D, Hong W, Fang Y, Qian Y, Yu S and $\mathrm{Li} \mathrm{H}$ : Alterations of microRNA-124 expression in peripheral blood mononuclear cells in pre- and post-treatment patients with major depressive disorder. J Psychiatr Res 78: 65-71, 2016.
47. Bahi A, Chandrasekar V and Dreyer JL: Selective lentiviral-mediated suppression of microRNA124a in the hippocampus evokes antidepressants-like effects in rats. Psychoneuroendocrinology 46: 78-87, 2014.

48. Cao MQ, Chen DH, Zhang CH and Wu ZZ: [Screening of specific microRNA in hippocampus of depression model rats and intervention effect of Chaihu Shugan San]. Zhongguo Zhong Yao Za Zhi 38: 1585-1589, 2013. (In Chinese).

49. Alimonti A, Carracedo A, Clohessy JG, Trotman LC, Nardella C, Egia A, Salmena L, Sampieri K, Haveman WJ, Brogi E, et al: Subtle variations in Pten dose determine cancer susceptibility. Nat Genet 42: 454-458, 2010.

50. Bosker FJ, Hartman CA, Nolte IM, Prins BP, Terpstra P, Posthuma D, van Veen T, Willemsen G, DeRijk RH, de Geus EJ, et al: Poor replication of candidate genes for major depressive disorder using genome-wide association data. Mol Psychiatry 16: 516-532, 2011.

51. Ferrari AJ, Charlson FJ, Norman RE, Flaxman AD, Patten SB, Vos $\mathrm{T}$ and Whiteford HA: The epidemiological modelling of major depressive disorder: Application for the Global Burden of Disease Study 2010. PLoS One 8: e69637, 2013.

52. Bocchio-Chiavetto L, Maffioletti E, Bettinsoli P, et al: Blood microRNA changes in depressed patients during antidepressant treatment. Eur Neuropsychopharmacol 23: 602-611, 2013.

53. Kempton MJ, Salvador Z, Munafò MR, Geddes JR, Simmons A, Frangou S and Williams SC: Structural neuroimaging studies in major depressive disorder. Meta-analysis and comparison with bipolar disorder. Arch Gen Psychiatry 68: 675-690, 2011.

54. Fan HM, Sun XY, Guo W, Zhong AF, Niu W, Zhao L, Dai YH, Guo ZM, Zhang LY and Lu J: Differential expression of microRNA in peripheral blood mononuclear cells as specific biomarker for major depressive disorder patients. J Psychiatr Res 59: 45-52, 2014. 